\title{
INITIAL RESULTS OF RF GUN EXPERIMENT
}

\author{
H. Abe, T. Asaka, H. Hanaki, A. Mizuno, S. Suzuki, T. Taniuchi*, K. Yanagida, \\ SPring-8, Hyogo 679-5198, JAPAN
}

\begin{abstract}
A photocathode RF gun was built and it has been operated for beam measurement at SPring-8. The cavity was tested by using high power RF up to $18 \mathrm{MW}$. The electric field gradient on the cathode reached $127 \mathrm{MW} / \mathrm{m}$. After RF conditioning, a laser pulse was irradiated on a copper cathode and the photo-emitted beam was accelerated up to 2.9 $\mathrm{MeV}$. An effective quantum efficiency of the cathode was obtained by changing laser power and field gradient.
\end{abstract}

\section{INTRODUCTION}

A photocathode RF gun is promising as an optional injector for the SPring-8 linac since a much lower emittance than that of the present thermionic gun system is expected. This feature can enable such future applications of the linac as a single-pass FEL based on SASE, which requires lower emittance and shorter bunch length.

For the optimization of a realistic gun system, an experimental and a simulation[1] study are being conducted in parallel. The purposes of this experiment were to develop a reliable simulation code by comparison with experimental results, to confirm stable operation of the RF cavity under a high gradient field environment, and establish the effectiveness of surface treatment for the reduction of dark current. Furthermore, technical problems with operating an RF gun system were revealed. In this paper, the initial results of the experiment are described.

\section{EXPERIMENTAL SET UP}

\subsection{Gun Cavity}

A single-cell RF cavity[2, 3] was designed by using MAFIA[4]. The field distribution of a single-cell cavity is considered to be simpler than that of multi-cell cavities and preferable for comparison with the simulation results. The accelerating gap length was determined by TS2 simulation to minimize the emittance, and the dimensions such as coupling hole size and cell radius were obtained by a 3D solver. A schematic drawing of the cavity is shown in Fig. 1.

A cavity wall made of OFHC copper was used as a photocathode. Two quartz windows for laser injection were located with an angle of $24^{\circ}$ from the cathode plane. Two couplers in this cavity were adopted to improve the field symmetry and shorten the filling time. The displacement of the field center from the geometrical center of the cavity was thus $0.13 \mathrm{~mm}$, while it was $0.55 \mathrm{~mm}$ for the single coupler case. By connecting a dummy load to the output

\footnotetext{
*Email: taniuchi@spring8.or.jp
}

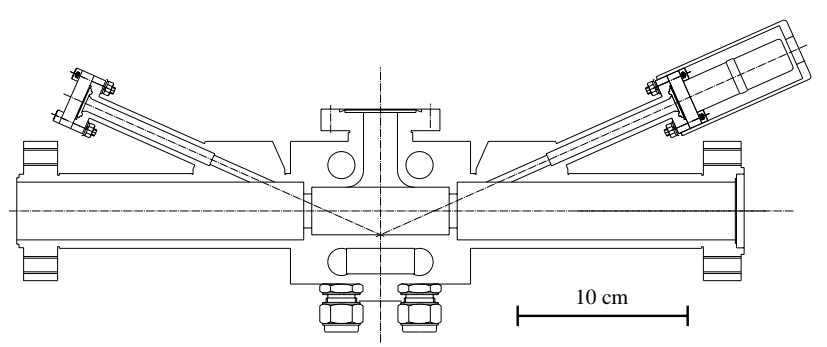

Figure 1: Schematic drawing of gun cavity.

coupler, the $\mathrm{Q}$ value of the cavity was reduced and the filling time was shortened. A shorter filling time enables a higher field gradient, more stable operation and reduction of dark currents. A higher gradient is needed to check the simulation results. The parameters of the cavity are summarized in Table 1.

Table 1: Parameters of high power model cavity.

\begin{tabular}{lcc}
\hline Frequency & $\mathrm{MHz}$ & 2856 \\
Number of cells & & Single \\
Accelerating gap & $\mathrm{mm}$ & 28 \\
Bore diameter & $\mathrm{mm}$ & 20 \\
Intrinsic Q value & & 13000 \\
External Q value for output port & & 3684 \\
External Q value for input port & & 2786 \\
Loaded Q value & & 1414 \\
Filling time & $\mu \mathrm{sec}$ & 0.31 \\
Shunt impedance (for $\beta=1)$ & $\mathrm{M} \Omega$ & 1.16 \\
Emax / Ecathode & & 1.09 \\
Laser injection angle & {$[\mathrm{deg}]$} & $90 / 24$ \\
\hline
\end{tabular}

\subsection{Laser}

The seed laser used in this experiment was a cw modelocked Nd:YLF laser (Lightwave Model 131) with a repetition rate of $178.5 \mathrm{MHz}$. A single IR pulse sliced by a Pockels cell was amplified by flash-lamp-pumped regenerative amplifiers at a repetition rate of $10 \mathrm{~Hz}$. Then fourth harmoinic photons were generated by two BBO crystals. A UV pulse with a wavelength of $262 \mathrm{~nm}$ and a pulse duration of $10 \mathrm{ps}$ was transferred into the radiation-shielded area and focused on the gun cathode. The maximum energy of the laser pulse is about $2 \mathrm{~mJ}$ at $262 \mathrm{~nm}$. 


\subsection{RF System}

A block diagram of the high power RF system is shown in Fig. 2. A $35 \mathrm{MW}$ klystron was installed at the RF gun test area located in the Machine Laboratory Building next to the $1 \mathrm{GeV}$ linac. RF power generated from the klystron is divided into two waveguides, and one is fed into the gun cavity while the other is fed into a dummy load. The RF power divider consists of a Magic Tee, a phase shifter and a $3 \mathrm{~dB}$ coupler and can divide power in an arbitrary ratio. There is an RF window between the waveguide system and the cavity. The vacuum pressure in the cavity is kept lower than $10^{-5} \mathrm{~Pa}$ by a $100 \mathrm{l} / \mathrm{sec}$ sputter ion pump. The RF power can be monitored through the directional couplers (DC1 5) shown in Fig. 2.

The klystron drive frequency of $2.856 \mathrm{GHz}$ is generated by a $178.5 \mathrm{MHz}$ RF signal from the seed laser.

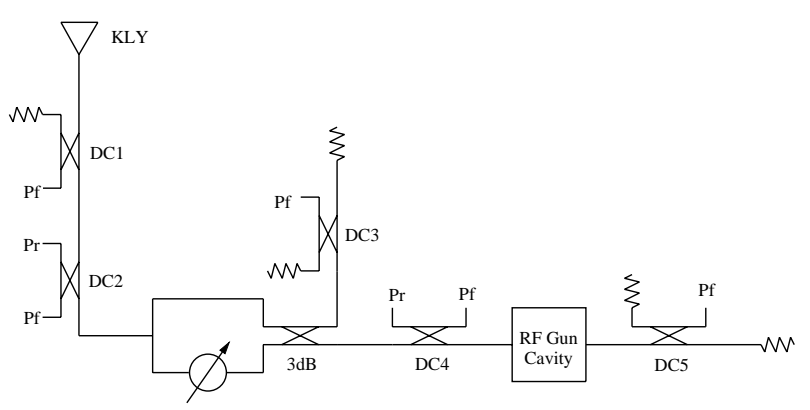

Figure 2: Block diagram of high power RF system.

\subsection{Beam Diagnostic System}

As shown in Fig. 3, the beam transport line consists of two solenoid magnets, two pairs of X-Y slits, a wall current monitor, two screen monitors, an energy analyzing magnet and a Faraday cup. All components are mounted on an optical table. We do not use a return yoke plate for the solenoid magnets, because the field distribution of such a coil can be solved easily and included into the simulation code. These magnets are only used to transport the beam with appropriate beam size but not optimized to minimize emittance. Two pairs of X-Y slits are used for emittance measurement.

During measurements mentioned below, it was found that the field distribution of solenoid magnets was distorted by the optical table made of magnetic material. As a result, a horizontal deflection of beam occurred. A steering magnet was installed to compensate the beam deflection due to the solenoid field. This optical table will be replaced by non-magnetic one.

\section{HIGH POWER TEST OF GUN CAVITY}

The RF conditioning of the cavity was performed with a pulse duration of $1 \mu \mathrm{sec}$ and a repetition rate of $10 \mathrm{~Hz}$. The vacuum in the cavity was kept lower than $1 \times 10^{-4} \mathrm{~Pa}$

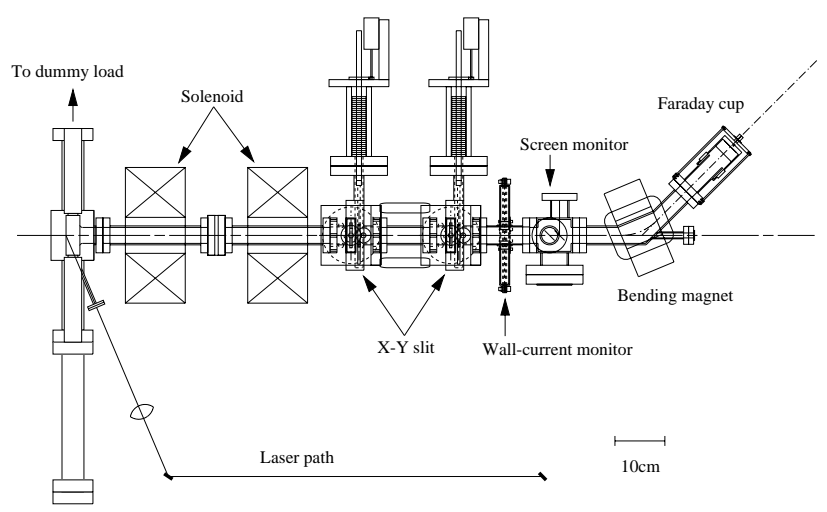

Figure 3: Top view of beam diagnostics system.

during the conditioning. The RF power fed into the cavity reached up to $18 \mathrm{MW}$ after 6 hours operation. The maximum RF power was limited due to klystron problems.

Fig. 4 shows typical RF wave forms at the upstream and downstream of the gun cavity. The electric field strength in the cavity saturated within $1 \mu \mathrm{sec}$, although some reflection effects could be seen on the wave forms due to the lack of an RF circulator. The pulse duration could be shortened during a single bunch mode to reduce dark currents and achieve more stable operation in a higher field gradient.

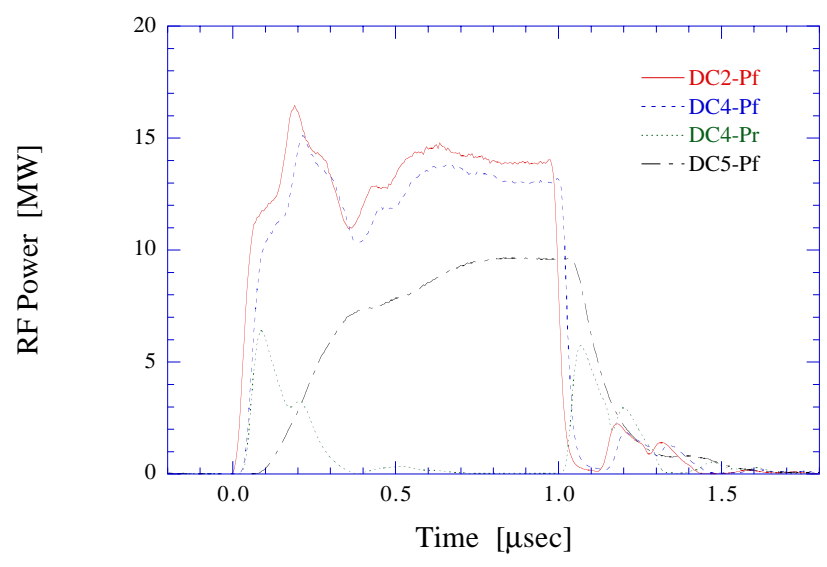

Figure 4: Typical RF waveforms obserbed by directional couplers shown in Fig. 2.

The dependence of the dark current on the field gradient is shown in Fig. 5. Dark currents were measured by using a Faraday cup directly connected to the cavity. The peak current increased exponentially with increasing the field gradient. It reached up to $10 \mathrm{~mA}$ when the field gradient was $121 \mathrm{MV} / \mathrm{m}$. The dark current will decrease largely along the transport line with solenoid fields.

Since continuous RF breakdowns and beam loading effects on reflection and transmitted RF power from the cavity could not be seen at this power level, the field gradient could be further increased. 


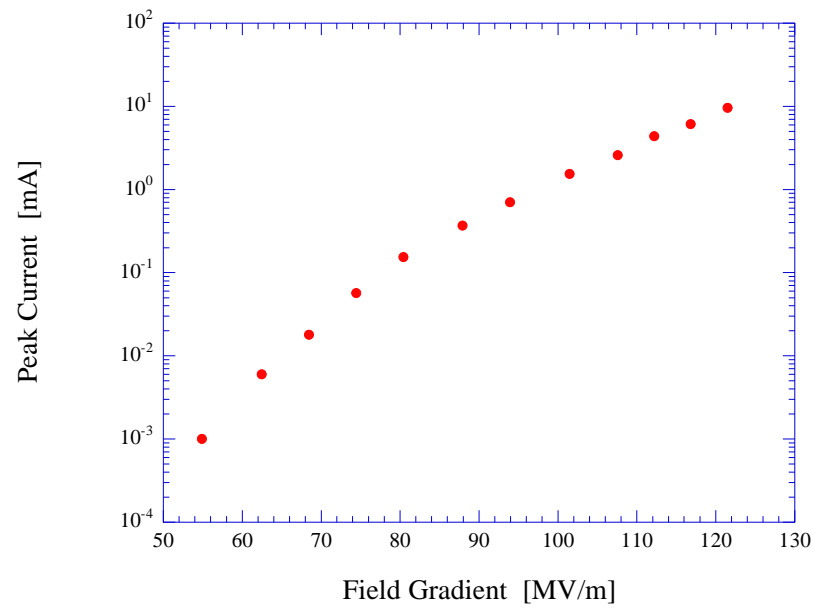

Figure 5: Dark current as a function of field gradient on cathode.

\section{BEAM MEASUREMENTS}

\subsection{Energy}

After RF conditioning of the cavity, a laser pulse was irradiated on the cathode and the photo-electrons were accelerated. The center beam energy was $2.6 \mathrm{MeV}$ and energy spread was about $\pm 10 \%$ when the input RF power was 18 MW. The field gradient on the cathode reached $127 \mathrm{MV} / \mathrm{m}$. These results agreed with the simulation results. A dependence of beam energy and energy spread on RF phase could not obtained because of an asymmetry of solenoid field.

\subsection{Quantum Efficiency}

The effective quantum efficiency was measured by connecting a Faraday cup directly to the cavity. A pulsed signal of the current was flattened by an RC filter with a time constant of 1.7 second. The contribution of dark currents was subtracted. Fig. 6 shows the bunch charge as a function of irradiated laser energy when the field gradients were 90 and $124 \mathrm{MV} / \mathrm{m}$. The RF phase was adjusted to obtain a maximum charge. From the slope of these data, the effective quantum efficiency for $90 \mathrm{MV} / \mathrm{m}$ case was found to be $2.4 \times 10^{-5}$. It was enhanced to $3.7 \times 10^{-5}$ in the case of $124 \mathrm{MV} / \mathrm{m}$ by Schottky effect. Though laser power was enough to produce a charge of more than $1 \mathrm{nC}$ at this condition, it could not possible to transport whole charge against the space charge force without solenoid magnets. In order to improve the quantum efficiency, a laser cleaning of the cathode and change of laser polarizing angle will be effective.

\section{CONCLUSIONS}

The fabricated gun cavity has shown the expected performance in experiments conducted so far. The dependences among the field gradient, charge, RF phase, laser power and so on should be further studied and compared with the

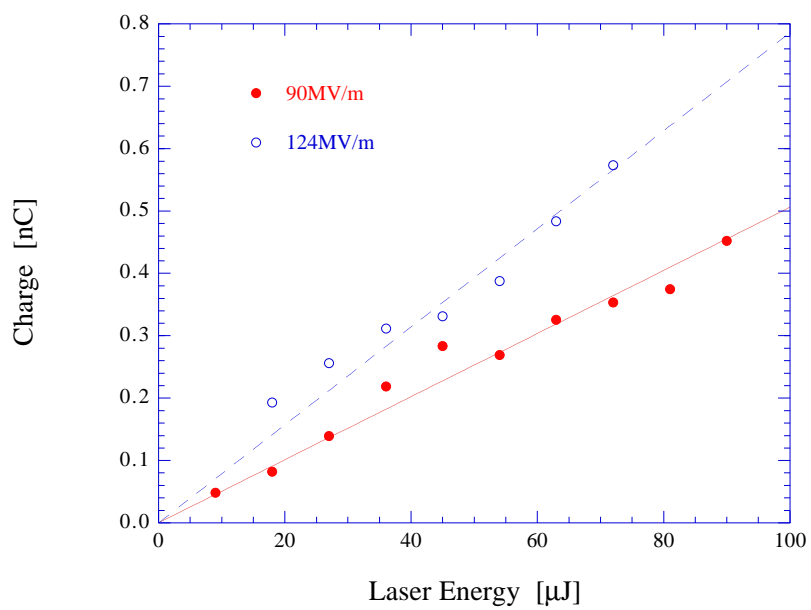

Figure 6: Beam charge per bunch as a function of irradiated laser energy.

simulation results. In order to investigate the beam characteristics in a higher field gradient, klystron power needs to be increased up to $35 \mathrm{MW}$. At this power level, the field gradient becomes about $180 \mathrm{MV} / \mathrm{m}$. Measurement of the transverse emittance and bunch length will be major activities in the next phase of this experiment. Furthermore, another two cavities will be evaluated to confirm the effectiveness of surface processing on the reduction of dark current.

\section{REFERENCES}

[1] A. Mizuno, et al., "Simulation for an rf gun test apparatus in the SPring-8 Linac", this conf., NY, (1999).

[2] T. Taniuchi, et al., "Study of an RF Gun for the SPring-8 Linac”, FEL conf., Rome, (1996).

[3] T. Taniuchi, et al., "Design of High Power Model Cavity for RF Gun”, FEL conf., Beijin, (1997).

[4] M. Bartsch et al., Computer Physics Communications 72 , 22-39 (1992). 